\title{
Experimental Models and Emerging \\ Hypotheses for Acute Lung Injury
}

Thomas R. Martin, MD*, Gustavo Matute-Bello, MD

\section{KEYWORDS}

- Lung injury • Animal model • Respiratory distress

Acute lung injury (ALI), and its more severe form, acute respiratory distress syndrome (ARDS), are syndromes of acute hypoxemic respiratory failure resulting from a variety of direct and indirect injuries to the gas exchange parenchyma of the lungs. The clinical syndrome is characterized by critical hypoxemia (partial pressure of oxygen in arterial blood/fraction of inspired oxygen $<300$ for ALI and $<200$ for ARDS), bilateral pulmonary infiltrates suggesting edema, no clinical or measured evidence of cardiac failure, and no other explanation for these findings. Pulmonary or nonpulmonary infections with sepsis are the most common causes of ALI and ARDS, although gastric aspiration, massive transfusions, trauma, and other factors contribute. ${ }^{1}$ The diversity of causes and the stereotyped physiologic and pathologic responses have made modeling human ALI and ARDS difficult, but new themes are evolving from experimental studies, some of which are reviewed in this article.

Most of what is known about the pathology of ALI and ARDS comes from the studies of patients who have died, although a limited number of patients have undergone open lung biopsy (Fig. 1). The pathologic changes include an early phase of diffuse alveolar damage, with an increase in endothelial permeability, evidence of intravascular thrombi, severe epithelial injury with denudation of alveolar wall basement membranes, accumulation of protein and fibrin-rich alveolar infiltrates in the airspaces, and abundant alveolar neutrophilic infiltrates. If patients survive, these changes progress for days to a repair phase, characterized by hyaline membrane formation, transition from neutrophilic to mononuclear infiltrates, and the appearance of intra-alveolar and

Supported in part by a research grant from the Department of Veterans Affairs and grants HL081764 (to TRM) and HL083044 (to GMB) from the National Institutes of Health.

Medical Research Service, Division of Pulmonary and Critical Care Medicine, Department of Medicine, VA Puget Sound Medical Center, University of Washington School of Medicine, Seattle, WA, USA

* Corresponding author. Hospital and Specialty Medicine, 111CHF, VA Puget Sound Medical Center, 1660 South Columbian Way, Seattle, WA 98108.

E-mail address: trmartin@u.washington.edu 

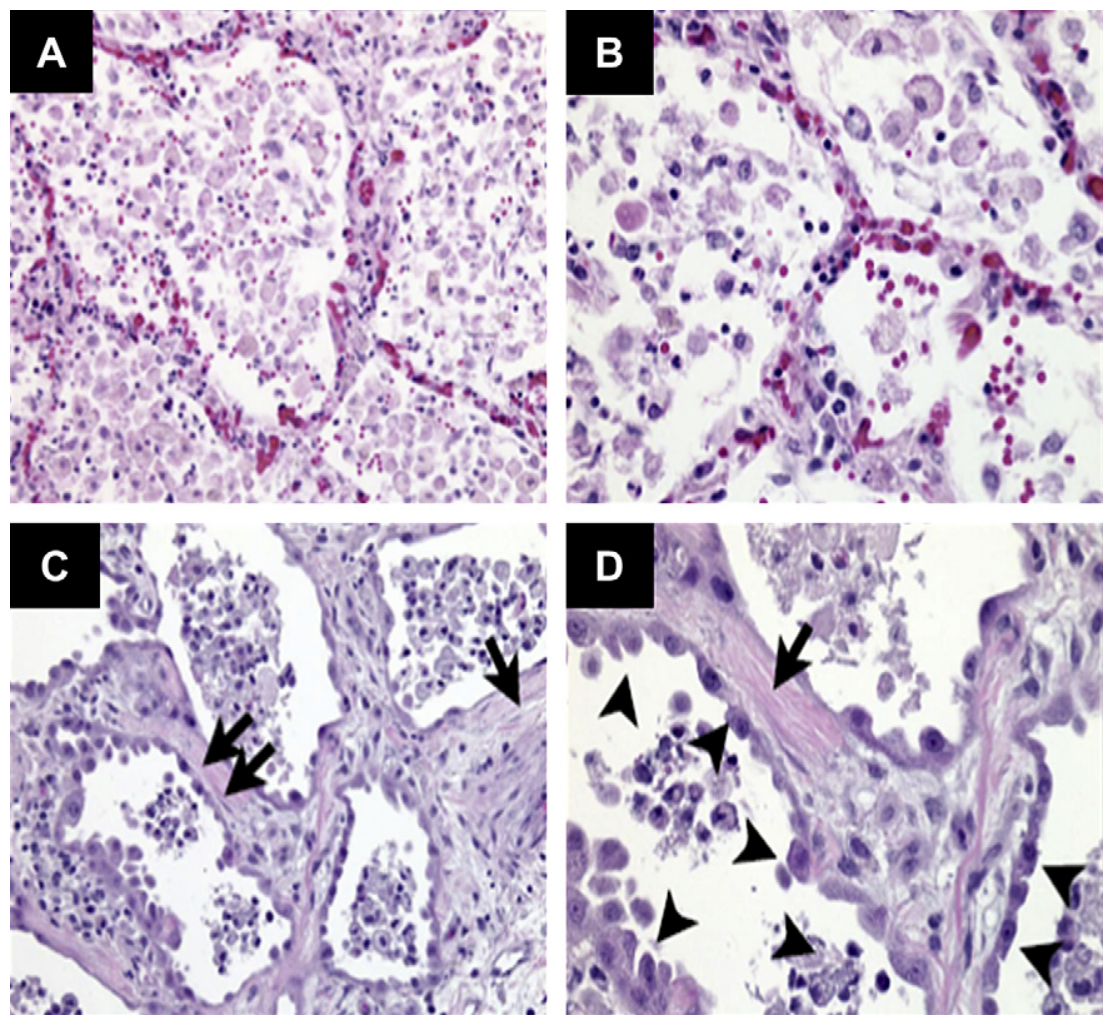

Fig. 1. ALI in humans. Photomicrographs of the lungs of 2 different patients with ALI, stained with hematoxylin and eosin. $(A, B)$ Acute phase. Alveolar spaces are filled with a mixed neutrophilic and monocytic infiltrate and alveolar wall capillaries are congested. Alveolar hemorrhage is visible. $(C, D)$ Later phase. Fibroproliferative response with collagen deposition in alveolar walls (arrows). Alveolar walls are lined with cuboidal epithelial cells that are proliferating type II pneumocytes. (From Matute-Bello G, Frevert CW, Martin TR. Animal models of acute lung injury. Am J Physiol Lung Cell Mol Physiol 2008;295:L381; with permission.)

interstitial fibrosis. Patients who survive often have persistent hypoxemia and restrictive ventilatory defects, but both of these abnormalities improve with time after hospital discharge. Neuromuscular weakness, rather than respiratory insufficiency, is the most important cause of long-term disability in survivors of ALI and ARDS. ${ }^{2,3}$

The causative factors that precipitate ALI can be grouped broadly into direct and indirect factors. Direct factors include bacterial and viral infections in the lungs and aspiration of gastric contents, all of which cause direct injury to the airway and alveolar epithelium and other structures in the airspaces. Indirect factors include systemic infections, which cause the sepsis syndrome, blood transfusions, and the effects of systemic medications and illicit drugs. This dual paradigm is plausible, even though clinical studies do not show major differences in outcomes in patients with direct versus indirect ALI.

A major overall theme emerging from clinical studies is that humans are inherently variable in their responses to the stimuli that cause ALI. Although investigators planning clinical trials strive to enroll uniform patient populations, clinicians recognize 
that patients with seemingly similar stimuli, for example pneumococcal bacteremia, vary a great deal in the clinical severity of their disease. Studies of how normal people respond to the common bacterial stimulus, gram-negative lipopolysaccharide (LPS), show differences of more than 2 orders of magnitude in cytokine responses in whole blood. ${ }^{4}$ Studies of normal humans who were high or low responders to bacterial products identified a polymorphism in the Toll-like receptor (TLR)1 that marked high cytokine responses to gram-positive bacterial peptidoglycan. ${ }^{5}$ This polymorphism was more common in critically ill patients with gram-positive sepsis who died. Thus, studying variability in innate immune responses in the normal population can provide important insights about disease susceptibility in critically ill patients.

\section{MODELING ARDS: THE ROLE OF ANIMAL MODELS}

Modeling the acute and chronic pathologic changes of ALI to understand the cellular and molecular pathogenesis has been a significant challenge from the time that ARDS was first described in humans. ${ }^{6,7}$ Many different animal models have been used and each has advantages and disadvantages. ${ }^{8}$ The ideal animal model would include an acute inflammatory response with an increase in microvascular and alveolar epithelial permeability, neutrophil influx into the alveolar spaces, and protein and fibrin-rich alveolar exudates in the acute phase. This response would be followed by an organization phase with an increase in alveolar mononuclear cells and interstitial lymphocytes, and a repair phase with proliferating type II pneumocytes and fibroblasts, and accumulation of interstitial and alveolar fibrin. These changes would be accompanied by acute hypoxemia and a decrease in lung compliance, along with measurable changes in systemic organ function. Ideally, the animal would be treated with mechanical ventilation to simulate the primary treatment applied to patients with ALI. These changes would evolve for several days, and surviving animals would be amenable to longerterm outcome studies to assess persistent changes in lung function and systemic organ function, particularly in the neuromuscular system.

Only large animal models permit studies in ventilated animals over time, such as ventilated and tracheostomized primates, dogs, sheep, or pigs. Such models are extremely expensive, because of the need to create an animal intensive care unit, and molecular reagents for large animals are limited. Short-term studies in mice, rats, and rabbits have been useful in studying individual pathways, but the ability to generalize results to humans is limited. ${ }^{8}$ Nevertheless, if the characteristics of the animal model are well known and the results are interpreted with appropriate caution, animal studies can provide focused evaluations of key physiologic and molecular pathways, and can be used to develop new hypotheses to test in humans.

Aside from size, important physiologic and immunologic differences exist among animal species (Table 1). Pulmonary intravascular macrophages (PIMs) are prominent in the pulmonary microcirculation of sheep, pigs, goats, cattle, and horses. In these animals, intravascular particles, including microbes, are more likely to localize in the pulmonary microcirculation and stimulate local intravascular inflammatory responses. Dogs, rodents, rabbits, nonhuman primates, and humans have few PIMs, and intravascular particles localize to macrophages in liver and spleen. ${ }^{9}$ Depletion of PIMs in sheep reduced lung injury from intravenous LPS. ${ }^{10}$ The nitric oxide (NO) pathway promotes vasodilation and microbial killing, and important species differences exist in NO production. ${ }^{11}$ Inducible nitric oxide synthase is prominent in rodents, and NO production is an important microbial killing mechanism in murine macrophages. Human macrophages produce far less NO unless they are suitably activated, typically by interferon- $\gamma \cdot{ }^{12-14}$ Nevertheless, the NO products, nitrate and nitrite, and evidence 


\begin{tabular}{|lllll|}
\hline $\begin{array}{l}\text { Table } 1 \\
\text { Unique characteristics of animal species relevant to modeling lung injury }\end{array}$ & \\
& $\begin{array}{l}\text { Identity with } \\
\text { Human } \\
\text { Animal }\end{array}$ & $\begin{array}{l}\text { Pulmonary } \\
\text { TLR4 HVR (\%) } \\
\text { Macrophages }\end{array}$ & LPS Sensitivity & $\begin{array}{l}\text { Nitric Oxide } \\
\text { Production }\end{array}$ \\
\hline Human & 100 & No & Intermediate & + \\
\hline NHP & 95 & No & Intermediate & + \\
\hline Pig & ND & Yes & High & ++ \\
\hline Dog & ND & No & Low & ++ \\
\hline Sheep & ND & Yes & High & ++ \\
\hline Rabbit & 57 & No & Intermediate & ++ \\
\hline Rat & 48 & No & Low & +++ \\
\hline Mouse & 48 & No & Low & +++ \\
\hline
\end{tabular}

Abbreviations: HVR, hypervariable region of TLR4; ND, not determined; NH, nonhuman primate.

From Matute-Bello G, Frevert CW, Martin TR. Animal models of acute lung injury. Am J Physiol Lung Cell Mol Physiol 2008;295:L381; with permission.

of nitration of intracellular proteins are detectable in the bronchoalveolar lavage fluid and alveolar macrophages of humans with $\mathrm{ALI},{ }^{15}$ suggesting that NO-dependent reactions are important in ALI. Bacterial recognition pathways via TLRs also differ among species, ${ }^{16}$ and divergent forms of TLR4 recognize different LPS structures, ${ }^{17}$ which could contribute to the known variation in LPS sensitivity among different species.

\section{EMERGING THEMES FROM ANIMAL MODELS AND EXPERIMENTAL STUDIES Interactions Between Stretch and Innate Immunity}

One important theme in clinical and experimental ALI is that activation of innate immunity adversely affects the lung's response to mechanical stretch. Patients with normal lungs, such as those with neuromuscular diseases, can be ventilated with large tidal volumes exceeding $10 \mathrm{~mL} / \mathrm{kg}$ without causing injury. Experimental studies show that, at normal tidal volumes, the alveolar walls in rodent lungs fold and unfold, whereas alveolar walls do not begin to stretch until lung volumes exceed about $40 \%$ of total lung capacity. ${ }^{18}$ In contrast, the effective alveolar volume of injured lungs is much lower than normal, owing to large areas of alveolar filling and collapse. In this case, the use of normal tidal volumes results in stretching of the walls of the open alveolar units. Experimental studies in a variety of systems show that activation of innate immunity pathways through TLR4 and other TLRs triggers acute inflammation and an increase in alveolar epithelial permeability. When human alveolar macrophages are exposed to cyclic pressure, cotreatment with LPS causes a marked accentuation of cytokine responses. ${ }^{19}$ Pretreatment of rats with intravenous LPS accentuated cytokine and inflammatory responses when the lungs were ventilated ex vivo. ${ }^{20}$ Mechanical ventilation and intravenous LPS have synergistic effects on lung inflammation at moderate tidal volumes via activation of complex transcriptional pathways. ${ }^{21-23}$ In addition to direct pulmonary effects, mechanical ventilation and intravenous LPS interact to cause systemic organ dysfunction, which is relevant for the pathogenesis of multiorgan failure. ${ }^{24}$ This seems to occur in part by enhancement of GADD45mediated signaling pathways in the lungs. ${ }^{22}$ The GADD45- $\gamma$ isoform activates a MAPK kinase kinase (MEKK4), leading to activation of p38 MAP kinase and Jun kinase (JNK), resulting in enhanced cytokine production. Mechanical stretch also causes upregulation of CD14 in rabbit lungs, and increased sensitivity of alveolar 
macrophages to LPS ex vivo. ${ }^{25}$ Because CD14 is a key coreceptor for LPS with TLR4, increased expression of CD14 provides a mechanism for synergy between LPS and larger tidal volume ventilation. In studies of ventilated mice, Smith and colleagues ${ }^{26}$ have found that this synergism between innate immunity and mechanical stretch seems to be acquired with age, because it does not occur in 3-week-old mice but is reproducibly present in 12-week-old mice.

Other activators of innate immunity are also present in the lungs of patients with ALI. A series of studies have shown that endogenous products generated by injury and inflammatory responses cause sterile inflammation when bacterial products are absent. ${ }^{27}$ These products, termed alarmins or danger-associated molecular patterns (DAMPs), include matrix molecules, hyaluronan, the nuclear protein HMGB1, oxidized phospholipids, and other factors that are present in normal lungs and released into the airspaces as a result of injury or inflammation. ${ }^{28-31}$ These endogenous products activate TLR4 and other TLRs, initiating inflammation in the same manner as LPS and other bacterial products. By implication, these endogenous molecules should also synergize with mechanical stretch to intensify injury in the lungs. One of the primary suggestions from this line of research is that interrupting the synergistic interactions between innate immunity and mechanical stretch in the lungs would be a strategy to limit the onset or severity of ALI in humans.

\section{The Fate of the Alveolar Epithelium in ALI}

Death of the alveolar epithelium in ALI can occur by either necrosis or apoptosis. The classic studies of Bachofen and Weibel ${ }^{32}$ examining lungs of patients who died with ALI showed evidence of widespread alveolar epithelial injury, in addition to alveolar hyaline membranes, microvascular injury, and thrombosis. Experimental studies have shown that high distending pressures caused by mechanical ventilation lead directly to disruption and necrosis of the alveolar epithelium in rats. ${ }^{33,34}$ In addition, type III bacterial exotoxins, such as pseudomonas ExoU and ExoS, cause direct lysis of the alveolar epithelium and other cells by attacking the cell membrane. ${ }^{35,36}$ Disruption of the alveolar epithelium by mechanical stretch can be treated by reducing the ventilator tidal volume, and is likely to explain, in part, the major success of the initial ARDS network trial of low-tidal-volume ventilation in ALI. ${ }^{37}$ Because necrosis cannot be regulated by manipulating cellular pathways, strategies to minimize necrosis must aim at prevention by lowering tidal volume and eradicating bacterial infection.

Apoptosis is a regulated form of cell death that has an essential role in development and repair. An important theme from experimental studies is that cell death pathways are activated in the lungs of patients with ALI and are likely to contribute to alveolar epithelial death. ${ }^{38}$ Apoptosis is mediated by a family of death receptors, principally the tumor necrosis factor (TNF) receptors (TNFR1 and TNFR2) and the Fas receptor. TNF $\alpha$ is not abundant in bronchoalveolar lavage (BAL) fluid of patients with ALI, and the concentrations of the soluble TNF receptors far exceeds the concentrations of free TNF $\alpha$, suggesting that TNF activity that exists is localized to lung tissues. ${ }^{39,40}$ The Fas receptor is present on the alveolar and airway epithelium, ${ }^{41}$ and biologically active soluble Fas ligand (sFasL) is detectable in the airspaces of patients with ALI. ${ }^{42,43}$ In experimental studies, activation of the Fas receptor in the lungs of mice causes alveolar epithelial apoptosis, and increased epithelial permeability and alveolar hemorrhage in rabbits. ${ }^{44,45}$ In mice and rabbits, activation of Fas also causes inflammation, with production of interleukin (IL)-8 and other acute inflammatory cytokines. Repeated activation of Fas in mice causes acute inflammation, an acute increase in alveolar epithelial permeability, and delayed fibrosis, which is dependent on macrophage metalloelastase, MMP-12. ${ }^{46}$ Studies with chimeric animals have shown that 
Fas on nonmyeloid cells of the lungs is required for apoptosis and inflammation to occur in response to Fas activation. ${ }^{47}$

The sFasL molecule is released from cell membranes via the action of membrane MMP-7. ${ }^{48,49}$ Like TNF $\alpha$, sFasL multimerizes in aqueous solution and the multimeric form clusters Fas receptors in the cell membrane. Clustered Fas molecules recruit caspase-8 molecules to the intracellular portions of the Fas molecules to form the death-inducing complex (DISC). Caspase-8 clusters are autocatalytic, yielding cleaved caspase-8, which initiates caspase cascades that lead to fragmentation of nuclear DNA and cellular apoptosis. The biologic activity of sFasL depends on the structure of the $\mathrm{N}$-terminal sequence of the molecule, and the state of aggregation. ${ }^{50}$ Oxidation of key methionine residues promotes aggregation of sFasL in solution and enhances biologic activity. Free MMP-7 cleaves the stalk region and reduces biologic activity, so that the intensity of the oxidizing environment and the concentration of soluble MMP-7 regulate the biologic activity of sFasL in vivo. ${ }^{50}$ Mice lacking an active Fas receptor (Ipr mice) have reduced lung inflammation when undergoing largevolume mechanical ventilation. Inactivation of Fas signaling in normal mice using siRNA technology reduced secondary lung injury in response to hemorrhagic shock and cecal ligation and puncture, suggesting that the Fas pathway in the lungs connects systemic responses with alveolar inflammation and epithelial injury. ${ }^{51,52}$

These and other data support the theme that Fas-mediated alveoli epithelial apoptosis is likely to be important in the acute lung injury process in humans, which in turn suggests that a strategy to inhibit apoptosis in the lungs might be useful in limiting the severity of $\mathrm{ALI}$ in humans. Apoptosis is also important in the resolution of injury, ${ }^{53}$ and tissue repair processes are initiated at the onset of ALI in humans, ${ }^{54,55}$ so any strategy modulating cellular apoptosis would have to be focused on the early phase of $A L I$ to avoid interfering with normal repair in the lungs.

\section{TGF $\beta$ as a Key Mediator of ALI}

Transforming growth factor $\beta$ (TGF $\beta$ ) is a pleuripotent cytokine that has a key role in tissue homeostasis. A latent form of TGF $\beta$ is activated when bound by the integrin $\alpha-v-\beta 6$ in lungs and skin. ${ }^{56}$ Mice lacking the $a-v-b 6$ integrin were protected from lung injury following intratracheal bleomycin, and mice treated with an anti-TGF $\beta$ construct were protected from lung injury caused by bleomycin or LPS. TGF $\beta$ enhanced epithelial permeability in vitro in part by depleting intracellular glutathione. ${ }^{57}$ A subsequent study showed that TGF $\beta 1$ reduced expression of the epithelial sodium channel $(\mathrm{ENaC})$, and reduced sodium and water transport across rat and human type II alveolar epithelial cells and reduced amiloride sensitive sodium transport in intact rat lungs at a low dose that did not affect alveolar epithelial permeability. ${ }^{58}$ These animal studies suggest that TGF $\beta$ activation in the lungs of patients with ALI could be a mechanism that contributes to epithelial injury and impairs sodium and water transport out of the alveolar spaces. Strategies to inhibit TGF $\beta$ transiently might be considered in humans with ALI.

\section{Networks and Complexity}

Animal models of ALI and ARDS have been used primarily to study single pathways involved in lung injury, but treatments designed to inhibit single pathways have been unsuccessful in patients with sepsis, as well as ALI. Advances in proteomics and genomics technologies have enabled investigators to appreciate the complexity of ALI in humans as well as in animal models. In humans, analysis of proteins in human BAL fluid shows the complexity of protein networks at the onset of $A L I$ and the changes that occur over time. ${ }^{59}$ Key nodes in these networks identify central proteins, 
which could provide targets for new treatments. In addition, proteomics analysis identified the unsuspected importance of the nonprotein, $\beta$-estradiol, as a node in major protein networks. Gene array technology illustrated the complexity of mRNA networks in a canine model of ventilator-induced lung injury. ${ }^{60}$ Genes involved in inflammation and immune responses, cell proliferation, adhesion, signaling, and apoptosis were activated in the lungs, and major regional differences were noted between dependent and nondependent areas. This approach provided additional support for the role of apoptosis pathways in ALI. Genomic approaches have also been used to study the complexity of transcriptional responses in mice treated with mechanical ventilation with or without systemic LPS. ${ }^{23}$ Integrating gene expression profiling with gene ontology and promoter analysis enabled the construction of a regulatory map of important processes in the lungs of ventilated animals in the presence or absence of LPS as a simultaneous activator of innate immunity (Fig. 2). Differentially expressed biologic modules included those related to defense responses, immune responses, and oxidoreductase activity. The gene regulatory network included transcription factors such as IFN-stimulated response element IRF-7 and Sp1 (Table 2). Studies such as these highlight the complexity of the lung responses in experimental animals with $A L I$, and set the stage for strategies that address multiple pathways simultaneously or sequentially in critically ill humans.

\section{New Understanding of Specific Risk Factors for ALI}

Animal models have provided a new understanding of several risk factors for ALI, including the pathogenesis of ALI following gastric aspiration and the transfusion of blood products, and the roles of chronic alcohol use and fever. Aspiration of gastric contents exposes the airway and alveolar environment to a complex mixture of acid, particulates, and oropharyngeal bacteria and bacterial products. The classic model of acid aspiration in animals involves intratracheal instillation of acid, typically $\mathrm{pH}$ 1.5. This acid causes ALI and inflammation with production of IL-8 and other proinflammatory cytokines. However, humans are routinely treated with $\mathrm{H}-2$ antagonists and/or proton pump inhibitors, so that the $\mathrm{pH}$ of gastric acid is typically much higher in patients, and less acidic solutions do not injure the lungs of animals. Bregeon and colleagues $^{61}$ sampled gastric juice from critically ill patients and studied proinflammatory activity using a validated target cell assay. The gastric juice from critically ill patients had more proinflammatory activity than was detected in gastric juice of ventilated control patients. The samples with high proinflammatory activity in vitro caused intense lung inflammation in the lungs of ventilated rabbits, which was dependent on $\mathrm{IL}-1 \beta$ activity in the gastric juice and independent of $\mathrm{pH}$ and particulate matter. This finding helps to explain the intense, and often transient, inflammatory responses associated with gastric aspiration in patients who are treated with antacid regimens.

Transfusions of red blood cells, platelets, and other high volume plasma blood products are known to be associated with transfusion reactions, which can lead to severe transfusion-associated lung injury (TRALI). ${ }^{62}$ Animal models show that a priming event, such as administration of intravenous or intratracheal LPS, is usually required for lung injury, consistent with the observation that TRALI is more common in humans with critical illness. Interactions between antibodies, leukocytes, and platelets are typically involved, and lipid mediators in plasma also have been implicated. In one model, passive infusion of anti-MHC-1 antibodies led to TRALI that was dependent on the Fc- $\gamma$ receptor, neutrophils, and platelets. ${ }^{63,64}$ Mice raised in a barrier facility were less susceptible, and pretreating the mice with either intravenous or intratracheal LPS restored susceptibility. Studies with chimeric mice showed that the functional TLR4 on leukocytes was required for this effect, which increases trapping of 


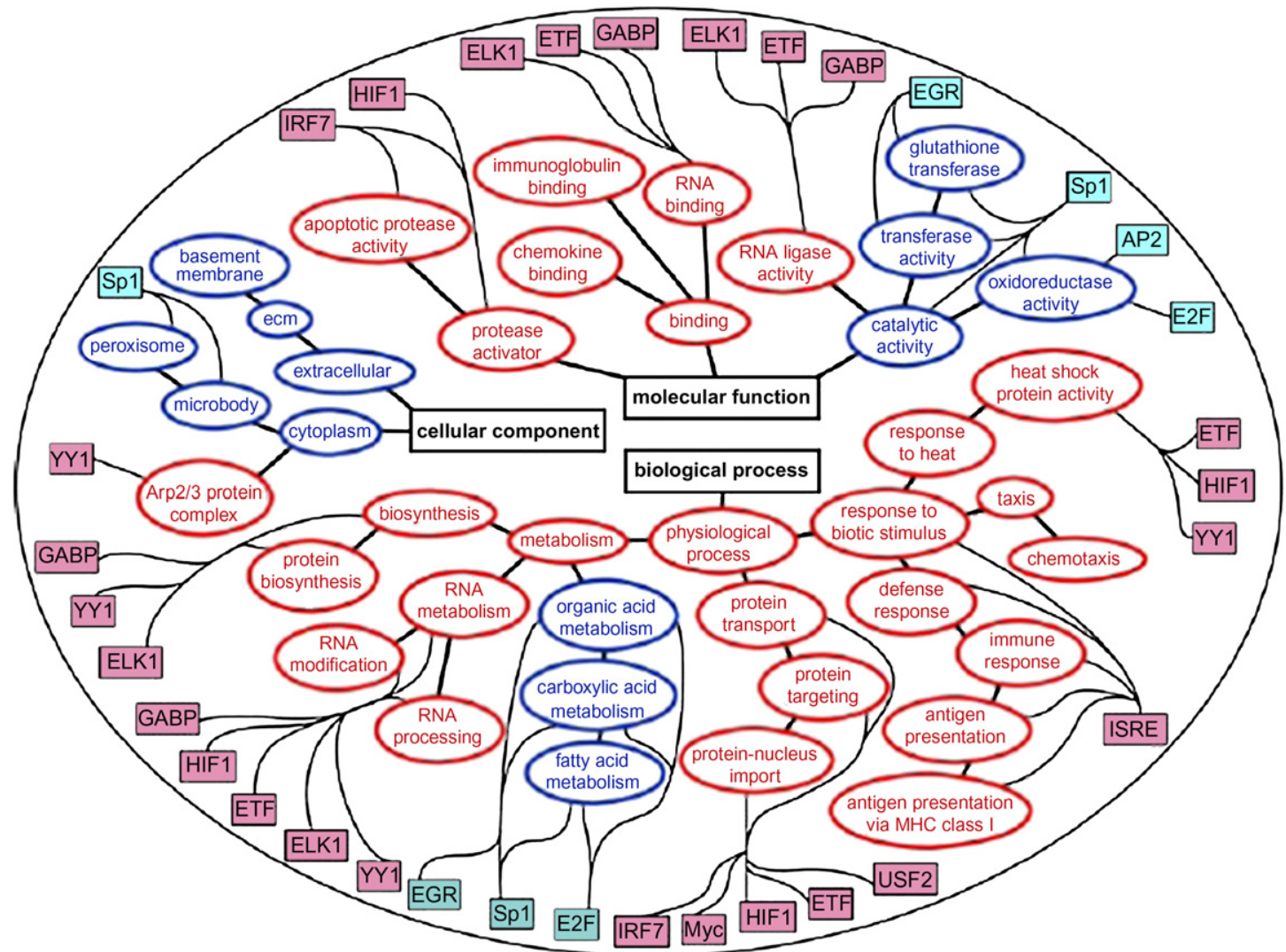

Fig. 2. Important processes and transcription factors identified during ALI in mice treated with intratracheal LPS and mechanical ventilation. Overrepresented biologic modules among upregulated (red ovals) and downregulated (blue ovals) genes are organized based on their gene ontology annotations and are assigned to 1 of 3 groups: molecular function, cellular components, and biologic processes. Putative transcription factors regulating genes within these modules are shown in the periphery. (From Gharib SA, Liles WC, Matute-Bello G, et al. Computational identification of key biologic modules and transcription factors in acute lung injury. Am J Respir Crit Care Med 2006;173:657; with permission.) 
polymorphonuclear leukocytes in the lung microcirculation and superoxide production in response to stimuli. Thus, there seems to be a key role for activation of innate immunity via TLR4, and perhaps other TLRs, in enhancing susceptibility to TRALI.

Animal and clinical studies have contributed to understanding the mechanisms by which chronic alcohol ingestion increases susceptibility to lung injury. ${ }^{65,66}$ Rats fed a high-alcohol diet (36\% of total calories) develop glutathione depletion in the epithelial lining fluid of the lungs and reduced sodium and water transport in vivo. ${ }^{67}$ Glutathione is a major intracellular pathway for capturing oxidant species, and glutathione depletion renders the lungs and other tissues susceptible to oxidative injury. Glutathione is depleted in type II pneumocytes from rats fed high-alcohol diets, and type II monolayers have increased permeability to high-molecular-weight solutes. ${ }^{68,69}$ These experimental observations parallel findings in clinically stable people who ingest alcohol on a chronic basis, who have reduced concentrations of glutathione in the alveolar epithelial lining fluid and have increased susceptibility to lung injury. ${ }^{70}$

Fever is a beneficial host response to bacterial and other infections, but many patients with ALI who are treated with antibiotic regimens do not have overt bacterial infection in the lungs. ${ }^{71}$ Fever improves outcome in mice with peritonitis and in other models of infection, ${ }^{72}$ but fever also worsens the response of the lungs to hyperoxia and localized klebsiella infections, in part by enhancing neutrophil recruitment. ${ }^{73,74}$ Lipke and colleagues ${ }^{75}$ found that fever has dramatic effects on innate immunity in the lungs, because the induction of fever in mice treated with low doses of LPS to stimulate TLR4 causes a dramatic increase in mortality, which is associated with the induction of apoptosis pathways in the lungs. These findings will drive better clinical studies of the effects of fever in patients who do not have major microbial infections.

\section{Stem Cells in Lung Injury}

One of the most interesting themes from animal studies is that mesenchymal stem cells can modulate ALI. Mesenchymal stem cells (MSC) are a population of progenitor cells with the ability to self-renew in an undifferentiated state and differentiate into mesenchymal tissues, such as bone, fat, smooth muscle, or collagen. ${ }^{76} \mathrm{MSC}$ have been known to exist in the mononuclear cell fraction of bone marrow, as defined by density gradient centrifugation. ${ }^{77}$ The International Society for Cellular Therapy has proposed the following criteria to define multipotent stromal mesenchymal cells: (1) adhesion to plastic; (2) expression of CD105, CD73, and CD90, and lack of expression of CD45, CD34, CD14 or CD11b, CD79a or CD19, and HLA-DR surface molecules; and (3) ability to differentiate into osteoblasts, adipocytes, and chondroblasts in vitro. ${ }^{78}$

Initial studies investigating the role of bone marrow-derived MSC (BM-MSC) in pulmonary fibrosis focused on the hypothesis that BM-MSC could be protective by regenerating injured lung tissue. In a seminal study, Ortiz and colleagues ${ }^{79}$ found that bleomycin-induced lung injury was decreased in C57BL/6 mice receiving intravenous injections of BM-MSC purified from bleomycin-resistant BALB/c mice, compared with mice receiving no BM-MSC. The protective effect occurred only when the BM-MSC were given immediately after the bleomycin challenge, but not when the cells were administered 7 days after bleomycin. Subsequent studies suggested that bleomycin induces mobilization of BM-MSC from the bone marrow, and possible migration into the lungs. ${ }^{80,81}$ Some of these studies suggest that the BMMSC engraft in the lungs and can differentiate into a variety of cell types. ${ }^{81}$ However, subsequent studies have shown that, although engraftment can occur, it is rare and the physiologic significance remains uncertain. ${ }^{82-84}$ Despite significant engraftment, MSC administration in a variety of injury models is associated with a decrease in the expression of several inflammatory cytokines, showing that the BM-MSC are 
Table 2

Enriched putative transcription factors among differentially expressed genes during mechanical ventilation (MV), LPS, and MV + LPS relative to untreated animals

\section{MV vs Control}

\section{Transcription Factor}

$P$ Value
Overrepresented Putative Transcription Factors Among Differentially Upregulated Genes

$$
\text { ETF }
$$

E2F

Nrf1

CREB

HIF1

-

Overrepresented Putative Transcription Factors Among

\begin{tabular}{|c|c|c|c|c|c|}
\hline Sp1 & $3.01 \times 10^{-6}$ & Sp1 & $8.38 \times 10^{-17}$ & Sp1 & $5.16 \times 10^{-26}$ \\
\hline NF-Y & $8.17 \times 10^{-5}$ & E2F & $1.96 \times 10^{-14}$ & E2F & $1.18 \times 10^{-21}$ \\
\hline$=$ & - & NF-Y & $1.96 \times 10^{-7}$ & EGR & $1.30 \times 10^{-9}$ \\
\hline$=$ & - & AP2 & $2.31 \times 10^{-6}$ & ZF5 & $1.78 \times 10^{-9}$ \\
\hline - & - & - & - & AP2 & $2.99 \times 10^{-9}$ \\
\hline- & - & - & - & NF-Y & $4.16 \times 10^{-9}$ \\
\hline
\end{tabular}

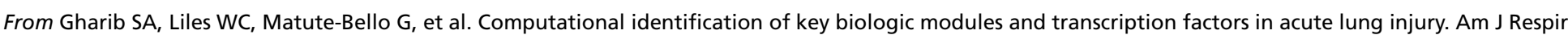
Crit Care Med 2006;173:656; with permission.

MV + LPS vs Control

\section{Transcription Factor}

$P$ Value

8.03

$5.36 \times 10^{-12} \quad$ CRel $\quad 8.51 \times 10^{-11} \quad$ ISRE

$1.12 \times 10^{-9} \quad \mathrm{IRF}$

$3.64 \times 10^{-8}$

$1.35 \times 10^{-6}$

$\mathrm{NF} \kappa \mathrm{B}$

$1.26 \times 10^{-9}$

$1.08 \times 10^{-7}$

$4.91 \times 10^{-6}$

-

-

$1.29 \times 10^{-11}$

SRE

$\mathrm{NF}-\mathrm{B}$ $2.48 \times 10^{-8}$ $3.84 \times 10^{-7}$

ICSBP

PU.1

CREB $8.01 \times 10^{-7}$

-

IRF-7

$1.92 \times 10^{-6}$

$2.05 \times 10^{-6}$

ifferentially Downregulated Genes 
able to modulate the inflammatory response. Gupta and colleagues ${ }^{85}$ confirmed the immunomodulatory properties of BM-MSC in vivo by finding that direct intratracheal instillation of BM-MSC attenuates LPS-induced lung injury by mechanisms involving a paracrine effect unrelated to tissue regeneration. Later studies by Ortiz and colleagues $^{86}$ suggested that the protective effect of BM-MSC on bleomycininduced lung injury is largely related to the ability of BM-MSC to release the IL-1 $\beta$ receptor antagonist (IL-1RA). Since then, several studies have shown that BMMSCs can attenuate injury in different experimental animal models. ${ }^{87}$ Thus, BMMSCs attenuate lung injury by immunomodulation, and most studies published thus far suggest a protective role. The role of MSC in lung inflammation and fibrosis is the subject of a separate review. ${ }^{88}$

\section{Resolution of $A L I$}

Human studies have shown that repair processes are initiated almost as soon as ALI begins. Markers of collagen production, reflecting activation of repair processes, are detectable at the onset of ALI. ${ }^{54,55} \mathrm{~A}$ great deal of work has been devoted to determining how neutrophils and their products are cleared from inflamed lungs. Isolated neutrophils rapidly undergo apoptosis in vitro, but the lung fluids of patients with ALI delay neutrophil apoptosis by a mechanism involving G-CSF and GM-CSF in lung fluids. ${ }^{89}$ Apoptotic neutrophils are rapidly ingested by macrophages in the airspaces, via recognition of phosphatidyl serine, calreticulin, and other structures expressed on the surface of apoptotic leukocytes. ${ }^{90-92}$ Neutrophil myeloperoxidase and other debris are identifiable in alveolar macrophages recovered from the BAL fluid of patients with ALI. ${ }^{89}$ The mechanisms that control the uptake and clearance of leukocytes and other cells undergoing necrosis or other nonapoptotic cell death are less well understood.

A new theme from animal studies is that lymphocytes also have an important role in the resolution of ALI. Studies with Rag- $1^{-1-}$ mice, which lack mature B and T cells, showed that resolution of LPS-induced lung inflammation was markedly delayed. ${ }^{93}$ Mortality was higher in the Rag-1 ${ }^{-1-}$ mice, and they remained clinically ill for a longer period of time than similarly treated C57BL/6 mice. Reconstitution of the Rag-1 ${ }^{-/-}$ mice with regulatory $\mathrm{T}$ cells expressing the IL-1 $\alpha$ receptor and the FoxP3 transcription factor (Tregs), improved the resolution of lung injury. Tregs increased with time after the onset of LPS-induced lung inflammation in normal mice, and transfer of Tregs into Rag-1 ${ }^{-/-}$mice increased lung levels of TGF $\beta$ and enhanced neutrophil apoptosis. Following these animal studies, the investigators found that Tregs were detectable by flow cytometry in lung lavage fluids of patients with ALI. Manipulation of regulatory $T$ cells might offer an approach to enhancing the repair of ALI.

\section{Viruses and $A L I$}

A consistent theme from animal studies is that the clinical manifestations of viral infections in the lungs reflect the primary sites of infection in the lungs. Adenoviruses infect primarily the airway epithelium via receptors on the basolateral surface of airway epithelial cells. This feature made replication-deficient adenoviral vectors attractive for gene therapy in the lungs. Adenoviral infections are characterized by bronchopneumonia, which can be severe, leading to acute respiratory failure. Studies in nonhuman primates showed that the severe acute respiratory syndrome (SARS) virus attacks alveolar type II cells, and SARS is associated with diffuse lung injury reflecting alveolar epithelial damage. ${ }^{94,95}$ By contrast, the hantavirus is found in lung microvascular endothelial cells and causes widespread lung edema soon after onset of the infection.

An additional theme from animal studies is that viral infections also enhance the sensitivity of the lungs to mechanical ventilation. Bem and colleagues ${ }^{96}$ found that 
mice infected with mouse pneumovirus to simulate respiratory syncytial virus (RSV) infection in children and then subjected to mechanical ventilation had much more severe lung inflammatory and injury responses than mice infected with pneumovirus alone, or mice treated with mechanical ventilation alone. The infected mice had increased cytokine production, increased alveolar epithelial permeability, and activation of apoptosis pathways. This suggests that the key treatment of children with severe RSV infection, mechanical ventilation, can worsen the response of the lungs to the underlying viral infection. Viruses stimulate innate immunity by interacting with TLR3 on the surface of macrophages and other cells. These and other findings support the conclusion that activation of innate immunity via several different TLRs has a synergistic effect with mechanical ventilation on lung injury.

\section{Lung Injury in Children}

One of the themes from clinical studies is that ALI is less frequent and less severe in children than adults even though mortality in unselected children with ALI is approximately $20 \% .^{1,97}$ Children have lungs that are still developing, and children typically have fewer comorbidities than adults with ALI. Nevertheless, a new theme from animal studies is that the interactions between the mechanical ventilator and the lungs of children might be different than in adults. Smith and colleagues ${ }^{26}$ compared the pulmonary responses of juvenile ( 3 weeks old, 5-7 g) and adult (16 weeks old, 25-30 g) mice in a model in which the mice were treated with intratracheal LPS, then subjected to mechanical ventilation for 2 or 4 hours. The adult mice had a synergistic increase in lung inflammation and protein permeability, as compared with animals treated with LPS alone, or mechanical ventilation alone. In contrast, a synergistic interaction between LPS treatment and mechanical ventilation was not found in the juvenile mice. This finding suggests that the adverse interactions between innate immunity and mechanical stretch increase with age. Microarray studies showed that there were major differences in clusters of genes activated in the juvenile and adult lungs in response to LPS and mechanical ventilation and suggested pathways that might be responsible for the different responses of juveniles and adults. Alvira and colleagues ${ }^{98}$ treated neonatal and adult mice with intraperitoneal LPS and found that lung inflammation and apoptosis occurred in adult but not neonatal mice. This finding was associated with persistent activation of NF- $\kappa \mathrm{B}$ p65/p50 heterodimers in the neonates, whereas in the adults there was initial activation

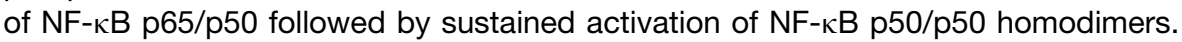
Developmental differences in NF- $\kappa \mathrm{B}$ activation could influence the severity or outcome of pulmonary infections, or the pulmonary response to mechanical ventilation. These studies comparing infant and adult animals could provide a much better perspective on the mechanisms that account for protection from ALI in children and increased susceptibility in adults.

\section{SUMMARY}

ALI is an important clinical problem that affects more than 200,000 people per year in the United States. Animal models have been useful in studying individual pathways involved in pathogenesis and new ideas for treatment. No single animal model mimics all of the clinical features of ALI in humans, and each animal model has unique features that affect responses to treatment. Nevertheless, many themes have emerged from animal models that provide valuable insight about lung injury in humans. Studies of innate immunity have shown that innate immunity is triggered not only by microbial products but also by endogenous byproducts of tissue damage and inflammation that can drive inflammation even in the absence of microbial 
products in tissue. Variability in host innate immune responses accounts for a great deal of variability in the clinical manifestations of ALI. Innate immunity and mechanical stretch have important synergistic interactions in adults that accentuate ALI. These synergistic interactions seem to be acquired with age and are much less pronounced in juvenile animals. Apoptosis pathways are important in clearance of bacteria from the lungs, and also in causing injury and death to alveolar epithelial cells, enhancing permeability edema. Animal models have highlighted the complexity of ALI in humans, by showing the multiplicity of pathways activated by microbial products, mechanical stretch, and the combination. Analysis of protein networks has identified unexpected components that link key protein pathways in the lungs. New light has been shed on clinical risk factors for ALI, such as gastric aspiration, blood product transfusion, alcohol excess, and fever. Stem cell biology has been extended to ALI with the finding of unexpected paracrine effects of MSC in reducing the severity of ALI. New ideas about the resolution of ALI have derived from studies of the clearance of apoptotic cells in the lungs, and the role of regulatory lymphocytes in recovery from lung inflammation and injury. Progress is being made, but strong links between the laboratory and the critical care bedside are still needed to translate new ideas from laboratory studies into clinical treatments that will lessen the severity and improve the outcome from ALI.

\section{REFERENCES}

1. Rubenfeld GD, Caldwell E, Peabody E, et al. Incidence and outcomes of acute lung injury. N Engl J Med 2005;353:1685-93.

2. Herridge MS, Cheung AM, Tansey CM, et al. One-year outcomes in survivors of the acute respiratory distress syndrome. N Engl J Med 2003;348:683-93.

3. Herridge MS, Tansey CM, Matte A, et al. Functional disability 5 years after acute respiratory distress syndrome. N Engl J Med 2011;364:1293-304.

4. Wurfel MM, Park WY, Radella F, et al. Identification of high and low responders to lipopolysaccharide in normal subjects: an unbiased approach to identify modulators of innate immunity. J Immunol 2005;175:2570-8.

5. Wurfel MM, Gordon AC, Holden TD, et al. Toll-like receptor 1 polymorphisms affect innate immune responses and outcomes in sepsis. Am J Respir Crit Care Med 2008;178:710-20.

6. Ashbaugh DG, Bigelow DB, Petty TL, et al. Acute respiratory distress in adults. Lancet 1967;2:319-23.

7. Petty TL, Ashbaugh DG. The adult respiratory distress syndrome. Clinical features and factors influencing prognosis and principles of management. Chest 1971;60:233-9.

8. Matute-Bello G, Frevert CW, Martin TR. Animal models of acute lung injury. Am J Physiol Lung Cell Mol Physiol 2008;295:L379-99.

9. Brain JD, Molina RM, deCamp MM, et al. Pulmonary intravascular macrophages: their contribution to the mononuclear phagocyte system in 13 species. Am J Physiol 1999;276:L146-54.

10. Sone Y, Serikov VB, Staub NC Sr. Intravascular macrophage depletion attenuates endotoxin lung injury in anesthetized sheep. J Appl Physiol 1999;87:1354-9.

11. Schneemann M, Schoedon G. Species differences in macrophage NO production are important. Nat Immunol 2002;3:102.

12. Schneemann M, Schoedon G, Hofer S, et al. Nitric oxide synthase is not a constituent of the antimicrobial armature of human mononuclear phagocytes. J Infect Dis 1993; 167:1358-63. 
13. Panaro MA, Acquafredda A, Lisi S, et al. Inducible nitric oxide synthase and nitric oxide production in Leishmania infantum-infected human macrophages stimulated with interferon-gamma and bacterial lipopolysaccharide. Int J Clin Lab Res 1999;29:122-7.

14. Nicolson S, da Gloria Bonecini-Almeida M, Lapa e Silva JR, et al. Inducible nitric oxide synthase in pulmonary alveolar macrophages from patients with tuberculosis. J Exp Med 1996;183:2293-302.

15. Sittipunt C, Steinberg KP, Ruzinski JT, et al. Nitric oxide and nitrotyrosine in the lungs of patients with acute respiratory distress syndrome. Am J Respir Crit Care Med 2001;163:503-10.

16. Rehli M. Of mice and men: species variations of Toll-like receptor expression. Trends Immunol 2002;23:375-8.

17. Hajjar AM, Ernst RK, Tsai JH, et al. Human Toll-like receptor 4 recognizes hostspecific LPS modifications. Nat Immunol 2002;3:354-9.

18. Tschumperlin DJ, Margulies SS. Alveolar epithelial surface area-volume relationship in isolated rat lungs. J Appl Physiol 1999;86:2026-33.

19. Pugin J, Dunn I, Jolliet $P$, et al. Activation of human macrophages by mechanical ventilation in vitro. Am J Physiol 1999;275:L1040-50.

20. Tremblay L, Valenza F, Ribeiro SP, et al. Injurious ventilatory strategies increase cytokines and cfos mRNA expression in an isolated rat lung model. J Clin Invest 1997;5:944-52.

21. Altemeier WA, Matute-Bello G, Frevert CW, et al. Mechanical ventilation with moderate tidal volumes synergistically increases lung cytokine response to systemic endotoxin. Am J Physiol Lung Cell Mol Physiol 2004;287:L533-42.

22. Altemeier WA, Matute-Bello G, Gharib SA, et al. Modulation of lipopolysaccharideinduced gene transcription and promotion of lung injury by mechanical ventilation. J Immunol 2005;175:3369-76.

23. Gharib SA, Liles WC, Matute-Bello G, et al. Computational identification of key biologic modules and transcription factors in acute lung injury. Am J Respir Crit Care Med 2006;173:653-8.

24. O'Mahony DS, Liles WC, Altemeier WA, et al. Mechanical ventilation interacts with endotoxemia to induce extrapulmonary organ dysfunction. Crit Care 2006;10:R136.

25. Moriyama K, Ishizaka A, Nakamura M, et al. Enhancement of the endotoxin recognition pathway by ventilation with a large tidal volume in rabbits. Am J Physiol Lung Cell Mol Physiol 2003;286(6):L1114-21.

26. Smith LS, Gharib SA, Frevert CW, et al. Effects of age on the synergistic interactions between lipopolysaccharide and mechanical ventilation in mice. Am J Respir Cell Mol Biol 2009;43(4):475-86.

27. Oppenheim JJ, Tewary P, de la Rosa G, et al. Alarmins initiate host defense. Adv Exp Med Biol 2007;601:185-94.

28. Noble PW, Jiang D. Matrix regulation of lung injury, inflammation, and repair: the role of innate immunity. Proc Am Thorac Soc 2006;3:401-4.

29. Abraham E, Arcaroli J, Carmody A, et al. HMG-1 as a mediator of acute lung inflammation. J Immunol 2000;165:2950-4.

30. Yu M, Wang $H$, Ding $A$, et al. HMGB1 signals through toll-like receptor (TLR) 4 and TLR2. Shock 2006;26:174-9.

31. Imai Y, Slutsky AS, Penninger JM. Identification of oxidative stress and Toll like receptor 4 signaling as a key pathway of acute lung injury. Cell 2008;133(2):235-49.

32. Bachofen A, Weibel ER. Structural alterations of lung parenchyma in the adult respiratory distress syndrome. Clin Chest Med 1982;3:35-56. 
33. Dreyfuss D, Saumon G. Ventilator-induced lung injury: lessons from experimental studies. Am J Respir Crit Care Med 1998;157:294-323.

34. Dreyfuss D, Soler P, Basset G, et al. High inflation pressure pulmonary edema. Respective effects of high airway pressure, high tidal volume, and positive end-expiratory pressure. Am Rev Respir Dis 1988;137:1159-64.

35. Kudoh I, Wiener-Kronish JP, Hashimoto W, et al. Exoproduct secretions of Pseudomonas aeruginosa strains influence severity of alveolar epithelial injury. Am J Physiol 1994;267:L551-6.

36. Kurahashi K, Kajikawa O, Sawa T, et al. Pathogenesis of septic shock in Pseudomonas aeruginosa pneumonia. J Clin Invest 1999;104:743-50.

37. NIH ARDSNet Group. Ventilation with lower tidal volumes as compared with traditional tidal volumes for acute lung injury and the acute respiratory distress syndrome. The Acute Respiratory Distress Syndrome Network. N Engl J Med 2000;342:1301-8.

38. Fine A, Janssen-Heininger $Y$, Soultanakis RP, et al. Apoptosis in lung pathophysiology. Am J Physiol Lung Cell Mol Physiol 2000;279:L423-7.

39. Park WY, Goodman RB, Steinberg KP, et al. Cytokine balance in the lungs of patients with acute respiratory distress syndrome. Am J Respir Crit Care Med 2001;164:1896-903.

40. Armstrong L, Thickett DR, Christie SJ, et al. Increased expression of functionally active membrane-associated tumor necrosis factor in acute respiratory distress syndrome. Am J Respir Cell Mol Biol 2000;22:68-74.

41. Fine A, Anderson NL, Rothstein TL, et al. Fas expression in pulmonary alveolar type II cells. Am J Physiol 1997;273:L64-71.

42. Matute-Bello G, Liles WC, Steinberg KP, et al. Soluble Fas-ligand induces epithelial cell apoptosis in humans with acute lung injury (ARDS). J Immunol 1999;163: 2217-25.

43. Albertine $\mathrm{KH}$, Soulier MF, Wang Z, et al. Fas and fas ligand are up-regulated in pulmonary edema fluid and lung tissue of patients with acute lung injury and the acute respiratory distress syndrome. Am J Pathol 2002;161:1783-96.

44. Matute-Bello G, Winn RK, Jonas M, et al. Activation of Fas (CD95) induces lung injury and apoptosis of type I and II pneumocytes in mice. Am J Respir Crit Care Med 1999;159:A697.

45. Matute-Bello G, Liles WC, Frevert CW, et al. Recombinant human Fas ligand induces alveolar epithelial cell apoptosis and lung injury in rabbits. Am J Physiol Lung Cell Mol Physiol 2001;281:L328-35.

46. Matute-Bello G, Wurfel MM, Lee JS, et al. Essential role of MMP-12 in Fasinduced lung fibrosis. Am J Respir Cell Mol Biol 2007;37:210-21.

47. Matute-Bello G, Lee JS, Liles WC, et al. Fas-mediated acute lung injury requires membrane Fas expression on non-myeloid cells of the lungs [abstract]. Am J Respir Crit Care Med 2004;169:A875.

48. Powell WC, Fingleton B, Wilson CL, et al. The metalloproteinase matrilysin proteolytically generates active soluble Fas ligand and potentiates epithelial cell apoptosis. Curr Biol 1999;9:1441-7.

49. Vargo-Gogola T, Crawford HC, Fingleton B, et al. Identification of novel matrix metalloproteinase-7 (matrilysin) cleavage sites in murine and human Fas ligand. Arch Biochem Biophys 2002;408:155-61.

50. Herrero R, Kajikawa O, Matute-Bello G, et al. The biological activity of FasL in human and mouse lungs is determined by the structure of its stalk region. $J$ Clin Invest 2011;121:1174-90. 
51. Perl M, Chung CS, Lomas-Neira J, et al. Silencing of Fas, but not caspase-8, in lung epithelial cells ameliorates pulmonary apoptosis, inflammation, and neutrophil influx after hemorrhagic shock and sepsis. Am J Pathol 2005;167:1545-59.

52. Perl M, Chung CS, Perl U, et al. Fas-induced pulmonary apoptosis and inflammation during indirect acute lung injury. Am J Respir Crit Care Med 2007;176:591-601.

53. Bardales RH, Xie SS, Schaefer RF, et al. Apoptosis is a major pathway responsible for the resolution of Type II pneumocytes in acute lung injury. Am J Pathol 1996;149:845-52.

54. Clark JG, Milberg JA, Steinberg KP, et al. Type III procollagen peptide in the adult respiratory distress syndrome: association of increased peptide levels in bronchoalveolar lavage fluid with increased risk for death. Ann Intern Med 1995; 122:17-23.

55. Chesnutt AN, Matthay MA, Tibayan FA, et al. Early detection of type III procollagen peptide in acute lung injury: pathogenetic and prognostic significance. Am J Respir Crit Care Med 1997;156:840-5.

56. Munger JS, Huang X, Kawakatsu $H$, et al. The integrin alpha $\vee$ beta 6 binds and activates latent TGF beta 1: a mechanism for regulating pulmonary inflammation and fibrosis. Cell 1999;96:319-28.

57. Pittet JF, Griffiths MJ, Geiser T, et al. TGF-beta is a critical mediator of acute lung injury. J Clin Invest 2001;107:1537-44.

58. Frank J, Roux J, Kawakatsu H, et al. Transforming growth factor-beta1 decreases expression of the epithelial sodium channel alphaENaC and alveolar epithelial vectorial sodium and fluid transport via an ERK1/2-dependent mechanism. J Biol Chem 2003;278:43939-50.

59. Chang DW, Hayashi S, Gharib SA, et al. Proteomic and computational analysis of bronchoalveolar proteins during the course of the acute respiratory distress syndrome. Am J Respir Crit Care Med 2008;178:701-9.

60. Simon BA, Easley RB, Grigoryev DN, et al. Microarray analysis of regional cellular responses to local mechanical stress in acute lung injury. Am J Physiol Lung Cell Mol Physiol 2006;291:L851-61.

61. Bregeon F, Papazian L, Delpierre S, et al. Role of proinflammatory activity contained in gastric juice from ICU patients to induce lung injury in a rabbit aspiration model. Crit Care Med 2008;36(12):3205-12.

62. Looney MR, Gilliss BM, Matthay MA. Pathophysiology of transfusion-related acute lung injury. Curr Opin Hematol 2010;17:418-23.

63. Looney MR, Su X, Van Ziffle JA, et al. Neutrophils and their Fc gamma receptors are essential in a mouse model of transfusion-related acute lung injury. J Clin Invest 2006;116:1615-23.

64. Looney MR, Nguyen JX, Hu Y, et al. Platelet depletion and aspirin treatment protect mice in a two-event model of transfusion-related acute lung injury. J Clin Invest 2009;119:3450-61.

65. Moss M, Parsons PE, Steinberg KP, et al. Chronic alcohol abuse is associated with an increased incidence of acute respiratory distress syndrome and severity of multiple organ dysfunction in patients with septic shock. Crit Care Med 2003; 31:869-77.

66. Joshi PC, Guidot DM. The alcoholic lung: epidemiology, pathophysiology, and potential therapies. Am J Physiol Lung Cell Mol Physiol 2007;292:L813-23.

67. Guidot DM, Modelska K, Lois M, et al. Ethanol ingestion via glutathione depletion impairs alveolar epithelial barrier function in rats. Am J Physiol Lung Cell Mol Physiol 2000;279:L127-35. 
68. Brown LA, Harris FL, Bechara R, et al. Effect of chronic ethanol ingestion on alveolar type II cell: glutathione and inflammatory mediator-induced apoptosis. Alcohol Clin Exp Res 2001;25:1078-85.

69. Brown LA, Harris FL, Guidot DM. Chronic ethanol ingestion potentiates TNFalpha-mediated oxidative stress and apoptosis in rat type II cells. Am J Physiol Lung Cell Mol Physiol 2001;281:L377-86.

70. Moss M, Guidot DM, Wong-Lambertina M, et al. The effects of chronic alcohol abuse on pulmonary glutathione homeostasis. Am J Respir Crit Care Med 2000;161:414-9.

71. Sutherland KR, Steinberg KP, Maunder RJ, et al. Pulmonary infection during the acute respiratory distress syndrome (ARDS). Am J Respir Crit Care Med 1995; 152:550-6.

72. Jiang $Q$, Cross AS, Singh IS, et al. Febrile core temperature is essential for optimal host defense in bacterial peritonitis. Infect Immun 2000;68:1265-70.

73. Hasday JD, Garrison A, Singh IS, et al. Febrile-range hyperthermia augments pulmonary neutrophil recruitment and amplifies pulmonary oxygen toxicity. Am J Pathol 2003;162:2005-17.

74. Rice P, Martin E, He JR, et al. Febrile-range hyperthermia augments neutrophil accumulation and enhances lung injury in experimental gram-negative bacterial pneumonia. J Immunol 2005;174:3676-85.

75. Lipke AB, Matute-Bello G, Herrero R, et al. Febrile-range hyperthermia augments lipopolysaccharide-induced lung injury by a mechanism of enhanced alveolar epithelial apoptosis. J Immunol 2010;184:3801-13.

76. Pereira RF, Halford KW, O'Hara MD, et al. Cultured adherent cells from marrow can serve as long-lasting precursor cells for bone, cartilage, and lung in irradiated mice. Proc Natl Acad Sci U S A 1995;92:4857-61.

77. Colter DC, Class R, DiGirolamo CM, et al. Rapid expansion of recycling stem cells in cultures of plastic-adherent cells from human bone marrow. Proc Natl Acad Sci U S A 2000;97:3213-8.

78. Dominici M, Le BK, Mueller I, et al. Minimal criteria for defining multipotent mesenchymal stromal cells. The International Society for Cellular Therapy position statement. Cytotherapy 2006;8:315-7.

79. Ortiz LA, Gambelli F, McBride C, et al. Mesenchymal stem cell engraftment in lung is enhanced in response to bleomycin exposure and ameliorates its fibrotic effects. Proc Natl Acad Sci U S A 2003;100:8407-11.

80. Xu J, Mora A, Shim H, et al. Role of the SDF-1/CXCR4 axis in the pathogenesis of lung injury and fibrosis. Am J Respir Cell Mol Biol 2007;37:291-9.

81. Rojas M, Xu J, Woods CR, et al. Bone marrow-derived mesenchymal stem cells in repair of the injured lung. Am J Respir Cell Mol Biol 2005;33:145-52.

82. Kotton DN, Fabian AJ, Mulligan RC. Failure of bone marrow to reconstitute lung epithelium. Am J Respir Cell Mol Biol 2005;33:328-34.

83. Loi R, Beckett T, Goncz KK, et al. Limited restoration of cystic fibrosis lung epithelium in vivo with adult bone marrow-derived cells. Am J Respir Crit Care Med 2006;173:171-9.

84. Sueblinvong V, Loi R, Eisenhauer PL, et al. Derivation of lung epithelium from human cord blood-derived mesenchymal stem cells. Am J Respir Crit Care Med 2008;177:701-11.

85. Gupta N, Su X, Popov B, et al. Intrapulmonary delivery of bone marrow-derived mesenchymal stem cells improves survival and attenuates endotoxin-induced acute lung injury in mice. J Immunol 2007;179:1855-63. 
86. Ortiz LA, Dutreil M, Fattman C, et al. Interleukin 1 receptor antagonist mediates the antiinflammatory and antifibrotic effect of mesenchymal stem cells during lung injury. Proc Natl Acad Sci U S A 2007;104:11002-7.

87. Sueblinvong V, Weiss DJ. Stem cells and cell therapy approaches in lung biology and diseases. Transl Res 2010;156:188-205.

88. Weiss DJ, Kolls JK, Ortiz LA, et al. Stem cells and cell therapies in lung biology and lung diseases. Proc Am Thorac Soc 2008;5:637-67.

89. Matute-Bello G, Liles WC, Radella F, et al. Modulation of neutrophil apoptosis by granulocyte colony-stimulating factor and granulocyte/macrophage colonystimulating factor during the course of acute respiratory distress syndrome. Crit Care Med 2000;28:1-7.

90. Henson PM, Tuder RM. Apoptosis in the lung: induction, clearance and detection. Am J Physiol Lung Cell Mol Physiol 2008;294(4):L601-11.

91. Gardai SJ, Bratton DL, Ogden CA, et al. Recognition ligands on apoptotic cells: a perspective. J Leukoc Biol 2006;79:896-903.

92. Nakanishi Y, Henson PM, Shiratsuchi A. Pattern recognition in phagocytic clearance of altered self. Adv Exp Med Biol 2009;653:129-38.

93. D'Alessio FR, Tsushima K, Aggarwal NR, et al. CD4+CD25+Foxp3 + Tregs resolve experimental lung injury in mice and are present in humans with acute lung injury. J Clin Invest 2009;119:2898-913.

94. Kuiken T, Fouchier RA, Schutten M, et al. Newly discovered coronavirus as the primary cause of severe acute respiratory syndrome. Lancet 2003;362:263-70.

95. Franks TJ, Chong PY, Chui $P$, et al. Lung pathology of severe acute respiratory syndrome (SARS): a study of 8 autopsy cases from Singapore. Hum Pathol 2003;34:743-8.

96. Bem RA, van Woensel JB, Bos AP, et al. Mechanical ventilation enhances lung inflammation and caspase activity in a model of mouse pneumovirus infection. Am J Physiol Lung Cell Mol Physiol 2009;296:L46-56.

97. Flori HR, Glidden DV, Rutherford GW, et al. Pediatric acute lung injury: prospective evaluation of risk factors associated with mortality. Am J Respir Crit Care Med 2005;171:995-1001.

98. Alvira CM, Abate A, Yang G, et al. Nuclear factor-kappaB activation in neonatal mouse lung protects against lipopolysaccharide-induced inflammation. Am J Respir Crit Care Med 2007; 175:805-15. 